\title{
The Flow-Based Market Coupling in Central Western Europe: concepts and definitions
}

Kenneth Van den Bergh, Jonas Boury, and Erik Delarue

TME WORKING PAPER - Energy and Environment Last update: September 2015

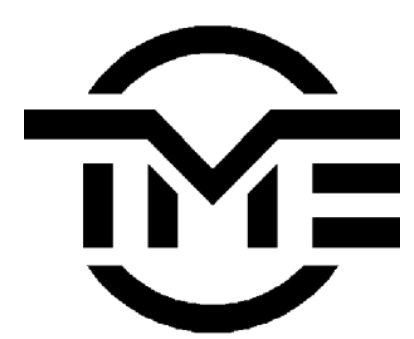

An electronic version of the paper may be downloaded from the TME website:

http://www.mech.kuleuven.be/tme/research/ 


\title{
The Flow-Based Market Coupling in Central Western Europe: concepts and definitions
}

\author{
Kenneth Van den Bergh, Jonas Boury and Erik Delarue'* \\ KU Leuven - Energy Institute, Celestijnenlaan 300 box 2421, 3001 Leuven, Belgium \\ *corresponding author: erik.delarue@kuleuven.be; tel. +3216 322 521; fax +3216 322985
}

\begin{abstract}
As of May $20^{\text {th }}$ 2015, Flow-Based Market Coupling is used for cross-border capacity allocation in Central Western European day-ahead markets (Belgium, the Netherlands, France and Germany/Luxembourg/Austria), replacing the Available Transfer Capacity method. This paper describes concepts and definitions of the Flow-Based Market Coupling. As such, this paper can serve as a starting point for further research on the methodology and market impact of Flow-Based Market Coupling.
\end{abstract}

Keywords. Cross-border Capacity Allocation; Flow-Based Market Coupling; Central Western European electricity market.

\section{Introduction}

The European Union strives for the completion of a liberalized single market for electric energy (Meeus et al., 2005; Batlle et al., 2014). The envisaged market design is referred to as the European Target Model for Electricity. The Target Model is an extensive and complicated set of proposals and network codes, see ENTSO-E (2015) for an overview, based on two broad principles: (1) energy-only regional markets and (2) market coupling between the different regional markets (Keay, 2013). Flow-Based Market Coupling (FBMC) is a mechanism to couple different electricity markets.

Coupling electricity markets increases the economic efficiency (Weber et al., 2010). However, the available transmission capacity between different market zones has to be taken into account properly. This is not straightforward since electricity doesn't flow directly from generator to consumer, but spreads out over all parallel paths in the network according to Kirchhoff's laws. There is hence a fundamental difference between commercial flows (i.e., the shortest path in the network between generator and consumer) and physical flows through the grid. As a consequence, the transmission capacity between two market zones cannot be fully allocated to commercial trade between these market zones since some of the capacity will be used by parallel flows resulting from trade between or within other market zones. The issue of allocating transmission capacity to the electricity market is referred to as cross-border capacity allocation.

Electricity markets in Europe are gradually integrating. A first major step was taken in November 2006 with the market coupling of the Belgian, Dutch and French day-ahead market (Powernext, 2006). This Trilateral Market Coupling was stepwise extended until the now-coupled area called Multi-Regional Coupling, covering 18 countries (Epexspot, 2015). ${ }^{1}$ Initially, the day-ahead market coupling was based on the Available Transfer Capacity (ATC) method for cross-border allocation, and it still is for most of the

\footnotetext{
${ }^{1}$ The market coupling covers Belgium, Denmark, Estonia, Finland, France, Germany/ Luxembourg/Austria, Great Britain, Latvia,
} Lithuania, the Netherlands, Norway, Poland (via the SwePol Link), Portugal, Slovenia, Spain and Sweden. 
market zones in the market coupling. However, since May $20^{\text {th }} 2015$, Flow-Based Market Coupling (FBMC) is being used for cross-border capacity allocation in Central Western European (CWE) day-ahead markets (i.e., Belgium, the Netherlands, France and Germany/Luxembourg/Austria) (Tennet, 2015).

The CWE FBMC was initiated in June 2007 with a Memorandum of Understanding between Energy Ministers, Transmission System Operators (TSOs), Power Exchanges (PXs), National Regulatory Authorities (NRAs), and Market Parties Platforms, stating the intention to implement FBMC in the CWE region (CREG, 2007). During 8 years, the FBMC methodology has been developed, evaluated and improved. This process is communicated in three important reports: the CWE FBMC feasibility report in 2011 (Amprion et al., 2011), the CWE FBMC intuitiveness report in 2013 (Amprion et al., 2013) and the CWE FBMC approval package in 2014 (Amprion et al., 2014). The FBMC methodology has been tested during 2 years with off-line parallel runs (2013-2014). These parallel runs have demonstrated a welfare increase in day-ahead compared to the ATC method, better price convergence between the different market zones and a good and sufficient level of reliability, stability and robustness of the FBMC system (CASC, 2015). Therefore, the National Regulatory Authorities approved the launch of the CWE FBMC in March 2015 (ACM et al., 2015).

The FBMC methodology is an important corner stone of the European Target Model. If the FBMC methodology turns out to be successful in the CWE day-ahead market, an extension to other market zones and markets (e.g., intra-day market) is likely. Despite its importance, the FBMC methodology is complex and poorly understood by market participants. A public consultation of the National Regulatory Authorities mentions that respondents assess their knowledge of the FBMC methodology as intermediate (ACM et al., 2014). Moreover, little literature on FBMC is publicly available. The FBMC methodology reports are the main source of information. Aguado et al. (2012) applied FBMC on historical order books and conclude that the transmission capacity provided by the FBMC methodology is larger than by the ATC methodology. As a result, social welfare and price convergence increase under FBMC. Waniek et al. (2010) came to similar conclusions, based on a model of the CWE region. Marien et al. (2013) show based on a simple model that the impact of various FBMC parameters on the market outcome is considerable. Based on this observation, they advocate for a transparent and adequate monitoring of the FBMC calculation process. Although insightful, none of the above mentioned literature presents a clear description of the FBMC methodology itself (partly because the FBMC methodology was still under construction at the time of writing).

This paper aims to present a clear and full description of the Flow-Based Market Coupling as implemented in the Central Western European day-ahead market. This paper can serve as a starting point for further research on the FBMC methodology, possible improvements and its impact on the market outcome.

The paper continues as follows. Section 2 discusses different methodologies for cross-border capacity allocation. Section 3 explains in detail the Flow-Based Market Coupling methodology as implemented in Central Western European day-ahead markets. Section 4 concludes and formulates policy recommendations. 


\section{Cross-border capacity allocation}

Electricity generators trade electricity with electricity retailers and large industrial consumers on Power Exchanges (PXs). ${ }^{2}$ The focus of this paper is on the day-ahead market, since FBMC is at the time of writing only implemented in the CWE day-ahead markets (market coupling in forward markets and intraday markets is still ATC-based). The power exchange collects generation and consumption bids and determines the optimal market outcome, i.e., the market outcome with maximum social welfare (consumer surplus, producer surplus and congestion rent). The objective function of the market clearing algorithm can be written in a simplified form as:

$$
\max _{x_{b, z}} \sum_{b, z} Q_{b, z} P_{b, z} x_{b, z}
$$

With $Q_{b, z}$ the quantity of bid $\mathrm{b}$ in market zone $\mathrm{z}$ in [MW] (generator bids are negative, consumption bids positive), $P_{b, z}$ the price of bid $b$ in market zone $z$ in [EUR/MWh], and $x_{b, z}$ the accepted share of bid $b$ in market zone $\mathrm{z}\left(0 \leq \mathrm{x}_{\mathrm{z}, \mathrm{b}} \leq 1\right)$.

The market outcome is subject to the market clearing condition, i.e., zonal generation equals zonal consumption plus net export:

$$
\sum_{b} Q_{b, z} x_{b, z}+N E X_{z}=0 \quad \forall z
$$

With NEX the Net Exchange Position of market zone $z$ in $[\mathrm{MW}$. A positive net exchange position indicates net export, a negative net import.

The market outcome is constrained by the available transmission capacity:

$$
\begin{aligned}
& -\overline{\bar{F}_{l} \leq F_{l} \leq \bar{F}_{l}} \quad \forall l \\
& F_{l}=f\left(N E X_{z}\right) \quad \forall l
\end{aligned}
$$

With $\bar{F}_{l}$ the maximum transmission capacity of line I available for the market in [MW], $F_{l}$ the flow through transmission line I, and $f$ a function linking the Net Exchange Positions with flows through the network. The market clearing algorithm (Eqs.1-4) is solved for every time step of the electricity market (e.g., for every hour in the European day-ahead electricity market). ${ }^{3}$ A detailed description of the market clearing algorithm used in the European day-ahead market can be found in (Price Coupling of Regions, 2013).

The cross-border capacity allocation consists of two sub-problems:

1. What is the transmission capacity available to the market (see $\bar{F}_{l}$ in Eq.3)?

2. What is the relationship between the Net Exchange Positions and flows through the grid (see $f$ in Eq.4)?

The fundamental difficulty with cross-border capacity allocation is that commercial flows (i.e., shortest path between generator and consumer) differ from physical flows (i.e., flows through all parallel paths according to Kirchhoff's laws). This is illustrated on Figure 1 where a commercial transaction between two nodes causes physical flows through the whole grid. Physical transmission constraints need to be

\footnotetext{
${ }^{2}$ Electricity can also be traded bilaterally, meaning that a generator and retailer/large consumer interact directly with each other and agree upon a trade contract.

${ }^{3}$ Different time steps can be linked to each other in the market clearing algorithm by complex bids such as block bids.
} 
translated into commercial transaction constraints or commercial transactions need to be translated into physical flows.

Different capacity allocation methods exist and are being used in electricity markets. The remainder of this section discusses three different methods: the nodal market clearing, the Available Transfer Capacity (ATC) method, and the Flow-Based Market Coupling (FBMC).

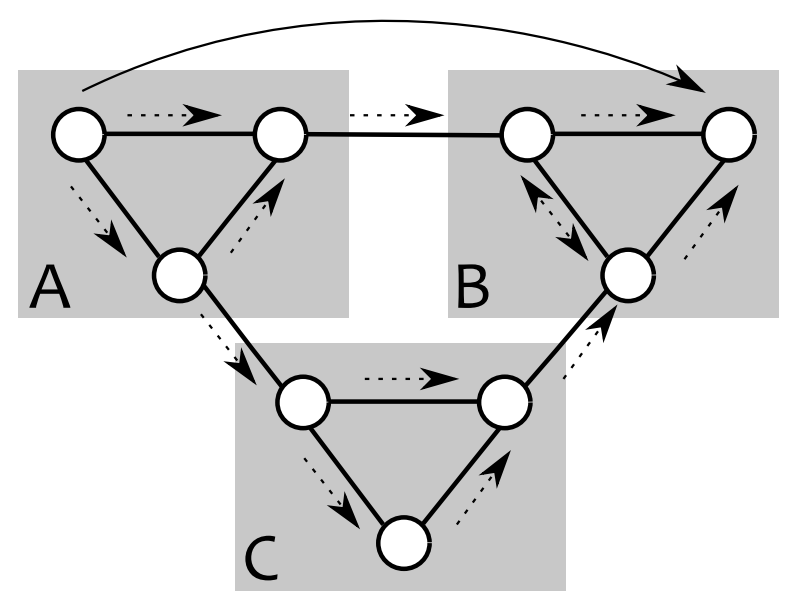

Figure 1. A commercial transaction between a node in market zone A and a node in market zone B (see solid arrow) results in physical flows in the whole grid (see dashed arrows).

\section{2.a) Nodal market clearing}

In an electricity market with nodal market clearing, all (relevant) physical transmission constraints are taken into account in the market clearing algorithm (Figure 2 shows the grid model of the running example in a nodal market clearing). As such, commercial transactions are correctly translated into physical flows and physical constraints are correctly accounted for in commercial transactions. In other words, capacity allocation happens simultaneously with the market clearing in a nodal market.

In a nodal market clearing, every node in the electricity grid is considered as one market zone. This implies that the size of the market clearing algorithm increases (the set $Z$ equals now the number of nodes in the electricity grid, i.e., every node can be considered as a separate market zone, and the set $\mathrm{L}$ contains all (critical) transmission lines) and that the Net Exchange Position equals the nodal grid injection $\left(\mathrm{NEX}_{\mathrm{z}}=\mathrm{P}_{\mathrm{n}}\right)$. The transmission constraints in the market clearing algorithm become:

$$
\begin{aligned}
& -F_{l}^{\max } \leq F_{l} \leq F_{l}^{\max } \quad \forall l \\
& F_{l}=\sum_{n} P T D F_{l, n}^{\text {nod }} P_{n} \quad \forall l
\end{aligned}
$$

with $F_{l}^{\max }$ the maximum allowable power flow on transmission line I in [MW], PTD $F_{l, n}^{\text {nod }}$ the nodal Power Transfer Distribution Factors and $P_{n}$ the nodal power injections. The maximum line capacity follows from the physical line capacity, reduced with a security margin to guarantee an $\mathrm{N}-1$ secure market outcome. The nodal PTDF gives the linear relationship between injections in the grid and flows through lines. The nodal PTDF results from a DC power flow analysis of the electricity grid. 
A nodal market clearing is implemented in several US electricity markets such as the PJM market (Hogan, 1999). Although nodal market clearing is based on a physical correct representation of the electricity grid, it is not part of the European Target Model for electricity. The European Target Model proposes a zonal market clearing with one uniform electricity price per zone.

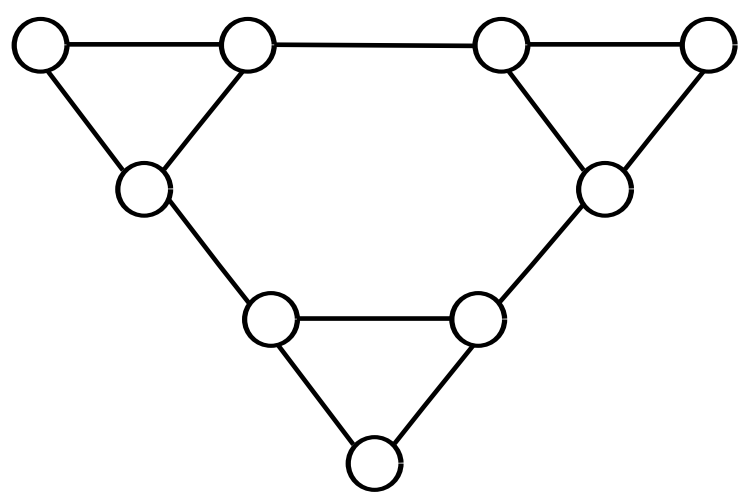

Figure 2. In a nodal market clearing, all (relevant) nodes and (critical) lines are taken into account in the market clearing algorithm. In this simple example, the nodal network consists of 9 nodes and 12 lines.

\section{2.b) Available Transfer Capacity}

In the Available Transfer Capacity (ATC) method, the link between commercial transactions and the physical characteristics of the electricity grid is strongly simplified. The nodes within a zone are grouped and replaced by an equivalent node, and only cross-border links are considered (see Figure 3a). In the ATC method, TSOs calculate the available capacity for the market $\bar{F}_{l}$ based on assumptions of the eventual market outcome and concomitant physical flows. In other words, capacity allocation takes place ex-ante the market clearing in the ATC-method.

The Available Transfer Capacity (ATC) is calculated as the maximum commercial exchange between two market areas, compatible with the physical transmission constraint and operational security standards (ETSO, 2001). The ATC of a cross-border link is independent of the flow on other cross-border links (see Figure $3 b$ ). In order to calculate the ATC, TSOs estimate the parallel flows that will result from the market outcome. The ATC calculation method is based on heuristic rules and day-2 estimations of the market outcome (i.e., the so-called Base Case). The ATC value is determined for each cross-border link (the set L equals the number of cross-border links now, no longer all transmission lines). The transmission constraints in the market clearing algorithm become:

$$
\begin{array}{ll}
A T C_{l}^{\text {min }} \leq F_{l} \leq A T C_{l}^{\text {max }} & \forall l \\
N E X_{z}=\sum_{l} A_{l, z} F_{l} & \forall z
\end{array}
$$

with $A T C_{l}^{\min }$ and $A T C_{l}^{\max }$ the ATC-value in the negative and positive direction, respectively, and $A_{l, z}$ the network incidence matrix. ATC-values can depend on the flow direction of the line due to the assumptions made in the ATC parameter calculation. The incidence matrix indicates whether a crossborder link is starting at a market zone $\left(A_{l, 2}=1\right)$, ending at a market zone $\left(A_{l, 2}=-1\right)$ or not connected to a market zone $\left(A_{1,2}=0\right)$. 
The ATC-market coupling is currently used in European electricity markets, except for the day-ahead market in Central Western Europe (which is using Flow-Based Market Coupling). Although the market clearing algorithm is rather simple with ATC market coupling, the calculation of the ATC-value itself is rather opaque and non-transparent for regulators. Moreover, the transmission capacity which is made available to the market is likely to be a conservative estimate of the physical available capacity. Given the strong assumptions inherent to the ATC method, the ATC value needs to be conservative to avoid physical line overloadings. Therefore, a new market coupling methodology is put in place in the CWE day-ahead market: the Flow-Based Market Coupling.

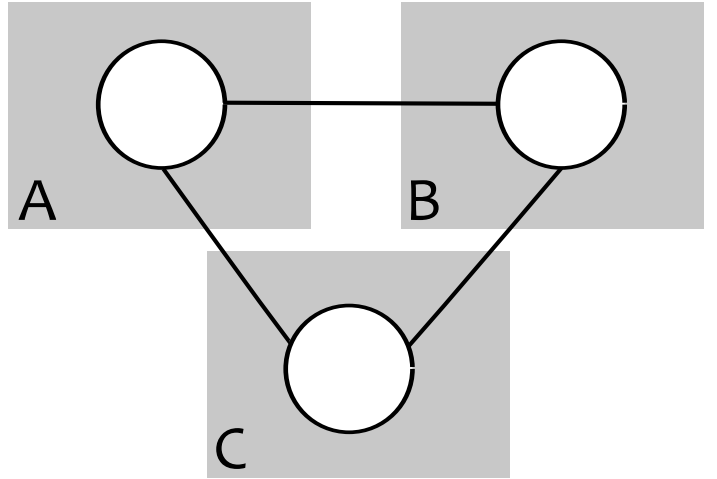

(a) Zonal market model.

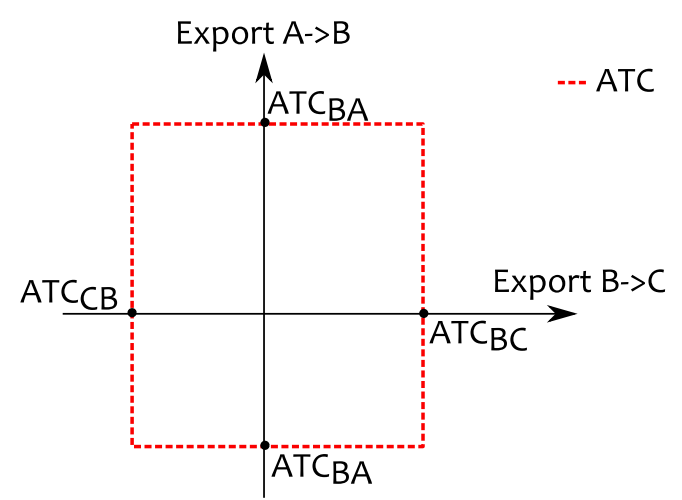

(b) Flow domain.

Figure 3. In the ATC method, only one equivalent node per zone is considered, with one cross-border link connecting the market zones. In this simple grid, the zonal network consists of 3 nodes and 3 cross-border links. The ATC flow domain is a rectangle, characterized by the ATC-values.

\section{2.c) Flow-Based Market Coupling}

Flow-Based Market Coupling (FBMC) can be seen as a combination of the zonal approach from the ATCmethod with the physical transmission constraints from the nodal market clearing. In FBMC, the physical transmission constraints are taken into account in the market clearing. However, since the zonal approach is retained, the grid constraints need to be simplified (see Figure 4). In other words, the capacity allocation in FBMC happens partly ex-ante the market clearing, and partly simultaneously with the market clearing.

In FBMC, all critical lines in the electricity grid are taken into account in the market clearing (the set L hence equals the number of critical lines, see subsection 3.b for more information on critical lines). ${ }^{4}$ The transmission constraints in the market clearing algorithm become:

$$
\begin{aligned}
& -R A M_{l} \leq F_{l} \leq R A M_{l} \quad \forall l \\
& F_{l}=\sum_{z} P T D F_{l, z}^{z o n} N E X_{z} \quad \forall l
\end{aligned}
$$

\footnotetext{
${ }^{4}$ Critical lines are considered in the $\mathrm{N}$-state and in critical N-1 states. Each index I refers to a combination of a critical line under a critical state, referred to as Critical Branch Critical Outage (CBCO). The size of the set $L$ is hence the multiplication of the number of critical lines and the number of critical N-1 states (plus one for the N-state).
} 
With $R A M_{l}$ the Remaining Available Margin of critical line I in [MW] and PTDF zon the zonal Power Transfer Distribution Factors. The zonal PTDF gives the linear relationship between Net Exchange Positions and flows through critical lines.

The RAM and the zonal PTDF are determined by the TSOs ex-ante the market clearing. These parameters are further referred to as the FBMC parameters and extensively discussed in section 3 . The FBMC parameters define the flow domain (see Figure 4). Each boundary of the FBMC flow domain refers to a critical line. The allowable commercial export/import between two market zones is no longer independent from the allowable commercial export/import between other market zones (unlike the ATC method). As the physical characteristics of the grid are better represented in the FBMC than in the ATC method, the FBMC parameters can be determined in a less conservative way. As a result, the FBMC flow domain is likely larger than the ATC flow domain.

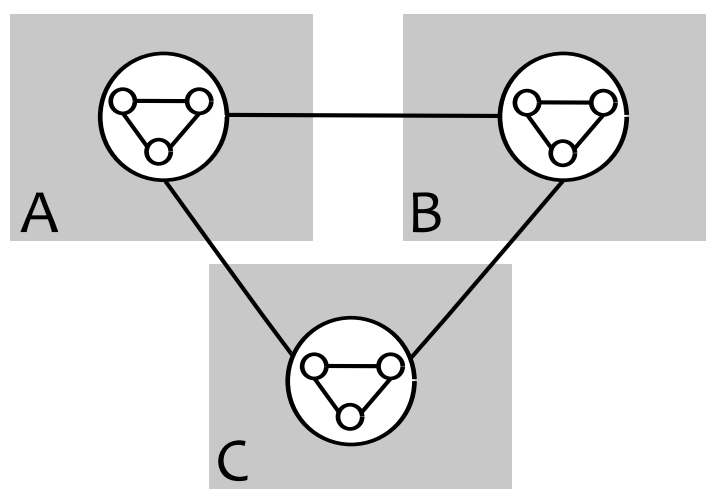

(a) Zonal market model.

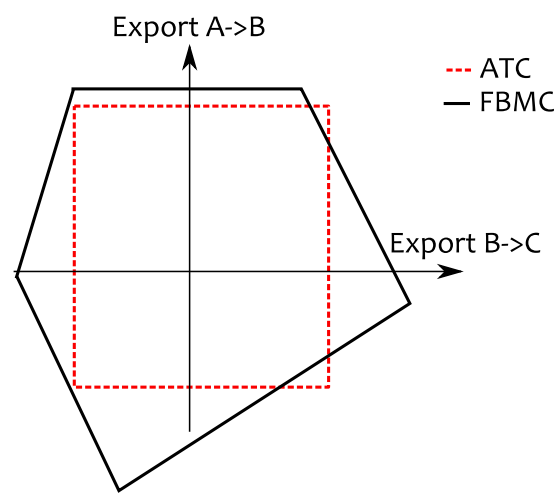

(b) Flow domain.

Figure 4. In the FMBC method, only one equivalent node per zone is considered, but all (critical) lines are taken into account. In this simple grid, the zonal network consists of 3 nodes and 12 lines. The FBMC flow domain is larger than the ATC flow domain as the physical characteristics of the grid are better represented in the FBMC method.

\section{Flow-Based Market Coupling parameters}

As mentioned in the previous section, two FBMC parameters are needed: the zonal Power Transfer Distribution Factors (PTDF ${ }^{20 n}$ ) and the Remaining Available Margin (RAM). In order to calculate both parameters, a so-called Base Case is needed.

The FBMC parameter calculation is started two days before the delivery day (i.e., D-2) and finished the morning day-ahead, so that they can be used in the day-ahead market clearing.

\section{3.a) Zonal Power Transfer Distribution Factors}

The zonal PTDF-matrix can be derived from the nodal PTDF-matrix by means of Generation Shift Keys (GSKs). GSKs give the nodal contribution to a change in zonal balance. For instance, $\mathrm{GSK}_{n, z}=0.3$ indicates that the generation at node $\mathrm{n}$ increases with $0.3 \mathrm{MW}$ if the zonal balance of zone $\mathrm{z}$ increases with $1 \mathrm{MW}$. The zonal PTDF-matrix can then be calculated as follows:

$$
\operatorname{PTDF}_{l, z}^{z o n}=\sum_{n} P T D F_{l, n}^{\text {nod }} G S K_{n, z} \quad \forall l, \forall z
$$




$$
G S K_{n . z}=\frac{d P_{n}}{d N E X_{z}} \quad \forall n, \forall z
$$

Eq.11 shows that the columns of the zonal PTDF-matrix are a weighted sum of the columns of the nodal PTDF-matrix, with the GSKs as weights.

The zonal PTDF is an approximation of the real physical characteristics of the grid. First, there is an inherent loss in accuracy by grouping nodes into zones. Information on the exact nodal injections in the grid is lost. Second, the GSKs are based on predictions of the market outcome, since the actual market outcome is not known yet. This implies that GSKs are subject to forecast errors. These two simplifications result in deviations between flows that are seen in the FBMC market clearing (i.e., flows based on the zonal PTDF) and the actual flows in the grid. Therefore, the transmission capacity available to the market is reduced with a safety margin to compensate for the approximations made by the zonal PTDF (see subsection 3.b).

In general, the GSKs include only power plants that are market-driven and flexible in changing the electric power output: coal fired plants, gas fired plants, oil fired plants, pumped storage units and conventional hydro units. ${ }^{5}$ Each TSO in the CWE region calculates the GSKs for its own control area. Different GSK methods are used by different TSOs. The Austrian TSO and each German TSO determines two GSK-matrices per day (peak and off-peak), based on reference days, after which the different GSKmatrices are combined into one GSK-matrix for the whole German/Luxembourg/Austrian market zone. Elia (Belgium) and TenneT NL (the Netherlands) determine one GSK-matrix per day, pro rata with predefined maximum and minimum production levels of power plants. RTE (France) determines a GSKmatrix for every hour, pro rata with generation in the base case.

\section{3.b) Remaining Available Margin}

The second FBMC parameter is the Remaining Available Margin (RAM). The RAM is the line capacity that can be used by the day-ahead market. The RAM procedure consists of two main steps. First, the critical branches and critical outages are determined (the set of lines I equals the number of critical branches under the critical outages). Second, the RAM is calculated for these critical branches under critical outages.

A critical branch is a transmission element (cross-border line, internal transmission line or transformer) which is significantly impacted by cross-border trade given an operational situation (normal $\mathrm{N}$-state or contingency cases such as an $\mathrm{N}-1$ state). A transmission line is considered to be significantly impacted if a zonal PTDF for that line is larger than $5 \%$. The critical branches are determined by each TSO for its own network. ${ }^{6}$ For each critical branch, the maximum allowable power flow $F_{l}^{\max }$ is determined as the physical (thermal) limit of the transmission element.

For each critical branch, the RAM is calculated as follows (the set $L$ refers now to the number of critical branches):

\footnotetext{
${ }^{5}$ Nuclear units are also taken into account in GSKs in France.

${ }^{6}$ TSOs have already significant experience with the determination of critical branches since it is also part of the ATC capacity allocation method.
} 


$$
R A M_{l}=F_{l}^{\max }-F_{l}^{r e f}-F A V_{l}-F R M_{l} \quad \forall l
$$

- $F_{l}^{\text {max }}$ the maximum allowable power flow on critical branch I in [MW];

- $F_{l}^{\text {ref }}$ is the reference flow on critical branch I in [MW] caused by commercial transactions outside the day-ahead power exchange (bilateral trades, forward markets, intra-day markets and real-time balancing). These commercial transactions can be internal (within a market zone) or external (between market zones); ${ }^{7}$

- $F A V_{l}$ is the Final Adjustment Value on critical branch I in [MW]. The FAV allows TSOs to take account of knowledge and experience that cannot be introduced in the formal FBMC method, such as an additional margin due to complex remedial actions, and can be positive or negative;

- $F R M_{l}$ is the Flow Reliability Margin on critical branch I in [MW]. The FRM is a safety margin that needs to compensate for approximations and simplifications made in the FBMC methodology such as the assumptions inherent to a zonal PTDF (see subsection 3.a), unintentional flow deviations due to load-frequency control, and the use of a linear grid model with a simplified topology.

The exact definition of the different flows in Eq.13 is confusingly and inconsistently formulated in the existing FBMC reports. Especially the definitions of $F_{l}^{\text {ref }}$ and $F R M_{l}$ are ambiguous, since some documents allocate part the reference flows to the Flow Reliability Margin (Amprion et al., 2014). More information on the calculation of the Remaining Available Margin can be found in Boury (2015).

\section{3.c) Base Case (Day-2 Congestion Forecast)}

The Base Case is a forecast of the state of the electricity system at moment of delivery, made two days before the delivery day. The Base Case is also referred to as the Day-2 Congestion Forecast (D-2CF). ${ }^{8}$ The Base Case is needed for (certain) GSK methods and to determine the reference flows in the RAM calculation.

The Base Case is determined in two main steps. First, every TSO estimates the local Base Case for its own control area. In a second step, the different local Base Cases are merged to one common Base Case.

Each TSO estimates its local Base Case based on a reference day. A reference day is a day for which the market outcome is known (i.e., a day in the past) with similar system conditions (e.g., weekday/weekend, winter/summer). The market outcome of the reference day is then updated with day-2 renewable generation forecasts, load forecasts and outage schedules for generation units and grid elements. In this first step, the TSOs coordinate the Net Exchange Positions of the reference day (in order to have a balanced CWE system), but each TSO applies a slightly different methodology to update the reference day. Note that the Base Case is also needed in the ATC capacity allocation method.

\footnotetext{
${ }^{7}$ The reference flow follows from $F_{l}^{r e f}=F_{l}^{b c}-\sum_{z} P T D F_{l, z}^{z o n} N E X_{z}$, with $F_{l}^{b c}$ the transmission flows in the Base Case in [MW].

${ }^{8}$ Besides a D-2CF, also a Day-Ahead Congestion Forecast (DACF) exists. The DACF is composed day-ahead in the evening (after the day-ahead market clearing) and is hence more precise than the D-2CF. The term 'Base Case' is sometimes used to refer to the D-2CF and sometimes to the DACF. In this paper, the term 'Base Case' always refers to the D-2CF.
} 


\section{3.d) Summary}

Two parameters need to be determined ex-ante the day-ahead market clearing: the zonal Power Transfer Distribution Factors (zonal PTDF) and the Remaining Available Margin (RAM). In order to calculate the zonal PTDF, Generation Shift Keys (GSK) are needed, which can follow from the Base Case. In order to calculate the RAM, the reference flows are needed, which can be derived from the Base Case.

The focus of this paper is on the FBMC methodology. However, the FBMC methodology is adjusted to account for several operational considerations. A backup procedure and fallback procedure are foreseen, in case of missing input data or the impossibility to calculate the FBMC parameters, respectively. After the FBMC parameters are calculated, a qualification and verification process takes place to check, amongst others, whether the Flow Based domain doesn't jeopardize grid security. Besides, TSOs can impose specific limitations to the allowable export/import in their network, so-called external constraints, in order to avoid market outcomes which lead to stability problems. A full description of these operational considerations can be found in Amprion et al. (2014). Finally, the FBMC methodology was adapted to avoid counter-intuitive flows (i.e., flows from a market zone with a high price to a market zone with a low price). This adapted version is referred to as the Intuitive Flow Based Market Coupling (Amprion et al., 2013).

\section{Conclusions and policy recommendations}

Flow-Based Market Coupling (FBMC) is in operation in the Central Western European day-ahead markets since May 20th 2015 (i.e., Belgium, the Netherlands, France and Germany/Austria). The FBMC replaced the Available Transfer Capacity (ATC) methodology. In FBMC, the allocation of transmission capacity is partly done simultaneously with the market clearing, unlike ATC where the allocation of transmission capacity is done ex-ante the market clearing. As such, more transmission capacity is made available to the market in FBMC. This is confirmed by off-line test runs of the FBMC, which indicate an increase in social welfare due to more transmission capacity, compared to ATC.

This paper presents FBMC in detail. It is clear that the methodology to calculate the FBMC parameters is complex. The complexity roots in the aim to combine a zonal approach with an improved physical representation of the grid. Due to this conflicting aims, several assumptions and approximations have to be made in the FBMC parameter calculation, making the methodology difficult to understand.

An often discussed issue regarding FBMC is transparency. Whether or not FBMC is a transparent crossborder capacity allocation mechanism is open for debate. On one hand, the FBMC methodology is defined more accurately and strictly than the ATC methodology, and as such regulating authorities can better supervise the FBMC parameter calculations (although the FBMC methodology leaves room for TSOs to steer the calculation process (Marien et al., 2013)). From a regulatory perspective, FBMC can thus be considered as more transparent than ATC. On the other hand, once the capacity allocation parameters are determined, an ATC-value indicates more clearly which transmission capacity is available to the market, compared to the FBMC parameters (zonal PTDF and RAM). Hence, from a market player's perspective, the transparency of FBMC can be questioned.

To end, the authors like to formulate three policy recommendations. First, the expected benefits of FBMC should be empirically evaluated based on historical market data (available as of May $20^{\text {th }}, 2015$ ). 
Second, coordination between TSOs should be improved further. With the introduction of the FBMC, bilateral coordination between TSOs (border-by-border, to determine the ATC-value) was replaced by regional coordination among all CWE TSOs. However, different TSOs still apply different methodologies in the FBMC process (e.g., Base Case determination, GSK calculation). Third, the performance of the FBMC depends on the market zone configuration. Smaller market zones allow a better representation of the physical characteristics of the grid, resulting in a better performance of the FBMC. In this respect, it might be useful to reconsider the market zone configuration in Europe.

\section{Acknowledgements}

The authors would like to thank the following persons for the information and insights they provided: $R$. Belmans and W. D'haeseleer (KU Leuven), A. Marien and P. Luickx (CREG), P. Reniers (Fluxys), $O$. Bronckart and J. Van Roost (Elia), and Y. Langer (Belpex). This paper presents the view of the authors, not the view of these persons. Any remaining errors are the sole responsibility of the authors.

\section{References}

ACM, Bundesnetzagentur, CRE, CREG, E-control and ILR. NRA public consultation on Flow Based Market Coupling: synthesis of respondent answers. 2014.

ACM, Bundesnetzagentur, CRE, CREG, E-control and ILR. Position Paper of CWE NRAs on Flow-Based Market Coupling. 2015.

Aguado M., R. Bourgeois, J. Bourmaud, J. Van Casteren, M. Ceratto, M. Jakel, B. Malfiet, C. Mestdag, P. Noury, M. Pool, and others. Flow-based market coupling in the Central Western European region: on the eve of implementation. CIGRE C5-204, 2012.

Amprion, APX-Endex, Belpex, Creos, Elia, EnBW, EpexSpot, RTE and Tennet. CWE Enhanced Flow-Based MC feasibility report. October 2011.

Amprion, APX, Belpex, Creos, Elia, Transnet BW, EpexSpot, RTE and Tennet. CWE Enhanced Flow-Based MC intuitiveness report. October 2013.

Amprion, APX, Belpex, Creos, Elia, EpexSpot, RTE, Tennet and Transnet BW. Documentation of the CWE FB MC solution: as basis for the formal approval-request, August 2014.

Batlle C., P. Mastropietro and R. Gómez-Elvira. Toward a fuller integration of the EU electricity market: physical or financial transmission rights? The Electricity Journal, 27.1: 8-17, 2014.

Boury J. Methods for the determination of flow-based capacity parameters: description, evaluation and improvements. KU Leuven Master Thesis, 2015.

CASC. CWE MC external parallel run. Available at www.casc.eu/en/Resource-center/CWE-Flow-BasedMC/Documentation, 2015.

CREG. Memoranding of Understanding of the Pentalateral Energy Forum on Market Coupling and Security of Supply in Central Western Europe. 2007.

Epexspot. Market Coupling - a major step towards market integration. 2015. 
ENTSO-E. Network Code Overview. Available at www.entsoe.eu/major-projects/network-codedevelopment, 2015.

ETSO. Definitions of Transfer Capacities in libelarised Electricity Markets. 2001.

Hogan W. Transmission congestion: the nodal-zonal debate revisited. Harvard University, John F. Kennedy School of Government, Center for Business and Government, 1999.

Keay M. The EU Target Model for Electricity Markets: fit for purpose? The Oxford Institute for Energy Studies, 2013.

Marien A., P. Luickx, A. Tirez and D. Woitrin. Importance of design parameters on flow-based market coupling implementation. $10^{\text {th }}$ International Conference on the European Energy Market, p.1-8, 2013

Meeus L., K. Purchala and R. Belmans. Development of the internal electricity market in Europe. The Electricity Journal, 18.6: 25-35, 2005.

Powernext. Everything you need to know about TLC. Available at static.epexspot.com/document/3830, 2006.

Price Coupling of Regions. Euphemia Public Description: PCR Market Coupling Algorithm, 2013.

Tennet. Flow-based methodology for CWE market coupling successfully launched. 2015.

Waniek D., C. Rehtanz and E. Handschin. Flow-based evaluation of congestions in the electric power transmission system. $7^{\text {th }}$ International Conference on the European Energy Market, p.1-6, 2010.

Weber A., D. Graeber, and A. Semmig. Market coupling and the CWE project. Zeitschrift für Energiewirtschaft, 34(4), p.303-309, 2010. 